\title{
PASSIVE IMMUNIZATION IN THE CLINICAL MANAGEMENT OF SECONDARY SPONTANEOUS RECURRENT MISCARRIAGE
}

Hanan A. Ghali, M.D.; Hoda A. Mansour, M.D.

\section{ABSTRACT}

Objective: To investigate whether intravenous immunoglobulin (IVIG) can inprove the chance of livebirth in women with a history of recurrent spontaneous miscarriage (RM).

Study design: Randomized, double-blind, placebo- controlled trial.

Patients and interventions: Fifty four women with a history of secondary (RM) were randomly assigned to receive doses of (IVIG) or placebo (Saline) starting as soon as pregnancy test was positive.

Results : The live birth rate (LBR) was significantly higher in the IVIG group when compared with placebo-infused group even after cxclusion of cases of ectopic pregnancy and cases with low scrum $\beta$ hCG and progesterone levels.

\section{INTRODUCTION}

Of all clinically detected pregnancies approximately $10 \%$ end in miscarriage (1). Recurrent spontaneous miscarriage (RM) is defined as a series of at least three pregnancy losses, a condition that affects $1 \%$ of women who desire a child (2) .RM is classified as either primary (never achieved a live birth) or secondary (normal pregnancy or pregnancy that progressed to at least 26 weeks followed by recurrent abortion) (3). The frequency of fetal aneuploidy decreases with the number of previous losses (4). On the Contraty, several studies have reported that the prevalence of immunological disturbances ${ }^{(5)}$ and of particular class I and class II HLA alleles (6,7) increases with the number of previous pregnancy losses.
A series of autoantibodies ${ }^{(8,9)}$ and a T-helper type I cytokine bias (10) can be found with increased prevalence in these patients as well. However, there is so far no definitive diagnostic test that can identify women who have such alloimmune dysfunction.

In patients negative for autoantibodies, active immunization with allogenic lymphocytes (ALT) from the partner (PLT) or third party donors (DLT) have been used.

ALT increases significantly the chance of livebirth among patients with primary RM with no auto-or allo-antibodies whereas no effect could be detected in patients with secondary RM (11), (12)

On the other hand, intravenous immunoglobulin (IVIG) has been used for several decades as treatment for a variety of immune-related diseases including; immune thrombocytopenic purpura, 
autoimmune neuropathies, systemic lupus erythematosus, Guillain-Barré syndrome, Kwasaki syndrome and others (13).

IVIG action may be mediated partially through induction of apoptosis in activated peripheral blood lymphocytes (14). Elevated levels of natural killer cells (NK) have been found in blood of women having miscarriages of karyotypically normal pregnancies (15).

Results of the seven placebo-controlled published studies using IVIG were conflicting with only two trials showing significant or almost significant treatment effect $(16,17)$. The remaining five trials, however showed no effect. (18-22).

The Cochrane meta-analysis reported no increase in livebirth after IVIG (23), but the updated meta-analysis of the trials showed that treatment may be efficient in patients with secondary $\mathrm{RM}^{(24)}$ unlike those with primary $\mathrm{RM}$.

This placebo-controlled study was carried out to determine the clinical outcome of pregnancy in patients with secondary RM with IVIG treatment during their current pregnancy.

\section{MATERIALS \& METHODS}

\section{Criteria of eligibility in this study :}

* History of $\geq 4$ confirmed miscarriages- with the present male partner- before the end of the twenty sixth gestational week, of which the last three had been consecutive.

* Normal uterine cavity as proved by hysterosalpingography or hysteroscopy.

* Normal parental chromosomes by G-band technique.

* Regular menestruation (cycle length 21-35 days).

* Positive pregnancy test.

* Absent endocrinological abnormality.

* Written informed consent.
No age restrictions were imposed allowing women up to 45 years of age to participate in this study. Pregnancies obtained by IVF or controlled ovarian hyperstimulation were excluded. None of the patients had immunoglobulin A (IgA) deficiency.

The presence of an autoimmune rheumatic disease was not a cause of exclusion in this study.

\section{Treatment protocol :}

Fifty four women mecting the above mentioned eligibility criteria were recruited for this study. Patients were instructed to conduct a pregnancy test if menstruation was three days overdue. Once pregnancy was confirmed, the first infusion was given.

After randomization, IVIG and equivalent amounts of saline (placebo) were administered. They could not be distinguished and the codes were blind for both the patients and the investigators. The typical infusion schedule was comprised of weekly infusions of $25 \mathrm{~g}$. IVIG from gestational week 5-9, and afterwards infusions were given fortnighily until gestational week 14 in women with only first trimester miscarriages and to gestational week 24 in women with second trimester losses, when no further infusions were given. The IVIG given in this study was GAMMARAAS $^{(\mathbb{1}}$ immunoglobulin IV $5 \%$ (human) manufactured by. Shanghai RAAS and packed by VACSERA; Egypt. The product is double virus inactivated by virus filtration and low $\mathrm{pH}$

\section{Measures of effect :}

The primary outcome measures were; (a) livebirth rate (LBR) after exclusion of patients with serum progesterone $<35 \mathrm{nmol} / \mathrm{L}$ and $\beta \mathrm{hCG}<100$ 
$U / L$ at the time of the first infusion, (b) LBR after exclusion of patients with ectopic pregnancies, (c) LBR after exclusion of a,b.

Secondary outcome measures were; gestational age at delivery, birth weight and Apgar scores. To have meaningful results 27 patients were assigned for each group. For each patient excluded or lost to follow up, one patient was added to maintain statistical power.

\section{Monitoring and laboratory tests:}

Transvaginal ultrasound scans were performed every second week from the 6 th till the $12^{\text {th }}$ gestational weeks to confirm the presence of heart beats and gestational age. Quantitative $\beta$ subunit of hCG was measured every week till week 10 . If the increase of $\beta \mathrm{hCG}$ was insufficient or vaginal bleeding occurred, weekly ultrasound examinations were undertaken. Survey by transabdominal ultrasound for congenital anomalies was done in gestational weeks 18 and 26 . After the $26^{\text {th }}$ week the routine pregnancy follow up was done.

Losses were considered fetal, if they occurred after the $10^{\text {th }}$ week; embryonic before the $10^{\text {th }}$ week but after documentation of fetal heart rate.
Pregnancies lost before depiction of fetal heart rate were pre-embryonic.

Checking for hepatitis $B$ antigens and antibodies against hepatitis $\mathrm{C}$ and HIV 1 and 2 was done on fresh samples obtained at the time of inclusion, in week 24 and 3 months after birth, or last infusion in case of miscarriage. Hepatitis $\mathrm{C}$ and $\mathrm{HIV}$ antibodies and $\mathrm{Hbs} \mathrm{Ag}$ virus were monitored using an enzyme immunoassay (EIA). Anticardiolipin antibodies (ACA) were measured using an enzyme linked immunosorbent assay (ELISA).

\section{Statistical analysis:}

Data were expressed as means $\pm \mathrm{SD}$. Paired-t-test and chi-square tests were used to compare results between IVIG and placebo groups.

\section{RESULTS}

From July 2000 to January 2004,54 women with a history of secondary recurrent miscarriages, who were confirmed to be pregnant before conclusion of the trial were randomized and included in this study.

Table I : Characteristics of patients at time of inclusion .

\begin{tabular}{|l|c|c|c|}
\hline & IVIG $(\mathbf{n}=\mathbf{2 7})$ & Placebo $(\mathbf{n}=\mathbf{2 7})$ & P \\
\hline Age (years) & $30.7 \pm 2.4$ & $31.8 \pm 1.1$ & NS \\
No with 4 previous losses & $10(37)$ & $13(48)$ & NS \\
No with 5 previous losses & $9(33)$ & $7(26)$ & NS \\
No with $\geq 6$ previous losses & $8(30)$ & $7(26)$ & NS \\
Anti cardiolipin positive & $9 / 27(33 \%)$ & $8 / 27(30 \%)$ & NS \\
Hormones at randomization & & & \\
$\quad *$ serum $\beta$ hCG (u/L) & $623.2 \pm 102$ & $549.5 \pm 133$ & NS \\
* serum progesterone (nmol/L) & $82.4 \pm 14$ & $76.3 \pm 12$ & NS \\
Body weight (Kg) & $72.7 \pm 16$ & $68.9 \pm 14$ & NS \\
\hline
\end{tabular}

NS = non significant

Data are presented as means \pm SD. 
All patients completed the infusion schedule until pregnancy was concluded by birth, or miscarriage.

Therapies used in management of RM e.g; progesterone, hCG, aspirin, heparin, prednisone, or allogenic lymphocyte immunization were not received by any patient during the course of this study. There was no statistical difference in the levels of $\beta$-hCG, and serum progesterone before the first infusion between IVIG and placebo groups (Table I).

Patients receiving IVIG achieved a LBR of
(21/27) $78 \%$ compared to $(9 / 2734 \%$ in placebo infused patients, $\mathrm{P}<0.01$ (Table II).

All pregnancy losses in IVIG group occurred in the first trimester (one fetal, one pre-embryonic, and two embryonic), whereas four miscarriages in the placebo group, occurred in the second trimester.

Seventy eight percent (7/9) of ACA positive patients assigned to IVIG group achieved a live birth. None of the ACA positive placebo patients had a live birth. Neonatal data did not differ between IVIG and placebo groups; (Table III).

Table II : Pregnancy outcomes in participant groups .

\begin{tabular}{|l|c|c|c|}
\hline \multicolumn{1}{|c|}{ LBR } & IVIG (n = 27) & Placebo (n= 27) & P \\
\hline Total $^{*}$ After Exclusions $^{*}$ & $21 / 27(78 \%)$ & $9 / 27(34 \%)$ & $<0.01$ \\
Appropriate hormones $^{\circ}$ & $21 / 25(84 \%)$ & $9 / 27(34 \%)$ & $<0.01$ \\
& $21 / 24(88 \%)$ & $9 / 23(39 \%)$ & $<0.01$ \\
\hline
\end{tabular}

* Exclusions of the 2 ectopic pregnancies in the IVIG group.

- Serum $\beta H C G>100 \mathrm{iu} / \mathrm{L}$ and serum progesterone $>35 \mathrm{nmol} / \mathrm{L}$ at first infusion.

Table III : Neonatal Data .

\begin{tabular}{|l|c|c|c|}
\hline & IVIG $(\mathbf{n}=\mathbf{2 7})$ & Placebo $(\mathbf{n}=\mathbf{2 7})$ & P \\
\hline Birthweight (g) & $3122.5 \pm 241$ & $3097.4 \pm 213$ & NS \\
Gestational age (wks) & $37.2 \pm 2.4$ & $38.1 \pm 1.3$ & NS \\
Apgar score $\leq 7$ at 5 min & 0 & 0 & NS \\
Cesarean section & 12 & 11 & NS \\
Neonatal disorders & 3 & 1 & NS \\
Sex of liveborn infants & $6 / 5 / 0$ & $3 / 6 / 0$ & P $<0.01$ \\
(male / female / unknown) & & & \\
\hline
\end{tabular}

Data are presented as means \pm SD. 
For neonatal abnormalities, in IVIG group two infants were born with alloimmune thrombocytopenia, which was corrected postpartum by giving IVIG to these infants and they were healthy thereafter.

Another infant in IVIG group had cleft palate. In the placebo group only one infant had talipes calcaneocavus.

Tests for antibodies against hepatitis $\mathrm{C}$ and HIV remained negative in all patients through and after pregnancy.

Few mild side effects viz; arthralgia, low grade fever, malaise, myalgias and local skin rash were noted in 13 patients receiving IVIG but they were mild in all patients.

Unexpectedly, there was a higher overall live-born female/male gender ratio which was significantly different from the 1:1 ratio.

\section{DISCUSSION}

The mechanisms that normally prevent a mother from rejecting her semi-allogenic conceptus are unclear.

The majority of recent research has focussed on immunologic causes but appropriate testing and treatment for RM due to immunologic causes is yet to be determined.

In 1988, Carreras et al. ${ }^{(25)}$ suggested that IVIG could be an alternative to leukocyte infusions in patients with RM.

IVIG is a highly purified human IgG prepared from pooled plasma with diverse antibody profile because thousands of donors contribute to the pool$^{(26)}$.

The use of IVIG to treat primary and secondary deficiencies in antibodies began 25 years ago.
Today, passive immunotherapy is attracting renewed interest as a new means to treat neurodegenerative, infectious, autoimmune and neoplastic diseases ${ }^{(27-29)}$.

Besides IVIG has been found to induce tolerance and improve clinical outcome after organ transplantation, and may work in the prevention of recurrent pregnancy loss ${ }^{(30)}$.

The results of uncontrolled pilot studies using IVIG were promising with successful pregnancy rate of $82 \%$ in women with $\mathrm{RM}^{(31-33)}$.

However, The results of the following five randomized controlled trials conducted to evaluate IVIG in RM were conflicting.

At a first glance, these diverging results provided no compelling reason to continue using IVIG in RM.

Nevertheless, the trials of immune therapy are too heterogenous in their inclusion of patients and treatment protocols to reach firm conclusions.

For instance, in this trial $58 \%(31 / 54)$ of patients had more than four previous miscarriages.

In the two placebo-controlled trials of the Danish group; 70-100\% of patients had $>4$ previous RM compared with only $19 \%{ }^{(18)}$ to $53 \%$ (20) of patients in other placebo controlled trials.

Based on the revised Cochrane meta-analysis showing improvement of livebirth only in patients with secondary RM; we selected only those patients in our study. A current literature search of RCTs using IVIG revealed that two trials were limited to women with primary RM (no live births) $(18,19)$, one involved only women with secondary $\mathrm{RM},{ }^{(16)}$ and two trials included both primary and secondary $\mathrm{RM}^{(17-20)}$.

There are many indications that women with at 
least four to five miscarriages and women with secondary RM have more immunological background for their problem (hence more likely to get IVIG therapeutic benefit) than those with fewer miscarriages and no previous live birth ${ }^{(34,35)}$.

Patients with IgA deficiency were excluded from this study. Similarly they were excluded from $4 \operatorname{RCTs}^{(16,19-20)}$.

The prevalence of $\operatorname{IgA}$ deficiency is 1 per 1000 and severe side effects are encountered, albeit rarely, in these patients when IVIG infusion is given to them; e.g.: nephrotoxicity, alopecia, aseptic meningitis and retinal necrosis ${ }^{(26)}$.

Regarding treatment protocols, IVIG infusion was started before conception in three trials (17), (36). (20), and during the first trimester in the present study and in three RCTs $(18),(16),(19)$.

To date there was no consensus regarding the dose employed as well. The doses given in RCTs varied widely, for example, Coulam et al. ${ }^{(17)}$ gave $500 \mathrm{mg} / \mathrm{kg}$ bw in follicular phase and $500 \mathrm{mg} / \mathrm{kg}$ bw / 4 weeks until 30 weeks of gestation, whereas, Christiansen et al. (16) gave $30-40 \mathrm{~g} /$ person in gestational weeks 5 and $6,20-30 \mathrm{~g} /$ person in gestational weeks 7,8 and every 2 weeks till the $4^{\text {th }}$ gestational week.

Shadowing experience with autoimmune thrombocytopenia Yamada and his coworkers (37) used a massive dose of $20 \mathrm{~g} / \mathrm{d}$ for 5 consecutive days, a total of $100 \mathrm{~g}$ during gestational weeks 4-7 usually between 4-5 wks gestation. The regimen of IVIG infusion adopted in this study was identical to that given by the Danish co-workers after the year $2000^{(24)}$.

Karyotypic analysis of abortuses was not done in this study since the more the number of previous losses the less the frequency of fetal aneuploidy ${ }^{(4)}$.
On the other hand, the live birth rate was significantly higher in IVIG vs placebo infused patients in this study unlike the insignificant difference in aggregate LBR of the 5 RCTs between the two groups.

Despite years of use, the mechanism of IVIG immunomodulation is still unclear.

It was recently shown ${ }^{(38)}$ that the protective effect of IVIG is due to up regulation of the inhibitory $\mathrm{Fc}_{\mathrm{C}}$ receptor $(\mathrm{Fc} \gamma \mathrm{RIIB}$ ) on macrophages in the reticuloendothelial system (RES).

In addition IVIG induces RES blockade (20). Moreover, IVIG use in immuno inflammatory conditions is associated with reduction in the serum concentrations of inflammatory markers such as IL- 6 and TNF $\alpha$ which are produced by macrophages and $\mathrm{T}^{-}$lymphocytes $\mathrm{CD} 4{ }^{(22)}$ and high serum TNF $\alpha$ levels were found in pregnant women with a history of RM ${ }^{(39)}$.

In this study, we measured serum creatinine in weeks 5 and 24 and at 3 months after birth or last infusion, to detect any renal troubles.

Acute renal failure is a rare IVIG complication with approximately 150 cases reported in literature. (20) It has been associated with diabetes, advanced age, preexisting renal disease and adminstration of intravenous immunoglobulin in sucrose-based solutions and rarely with maltose stabilized preparations (40). Sucrose, maltose, sorbitol, glycine, glucose or albumin are used as stabilizing agents to prevent immunoglobulin aggregation.

In this study we used GAMMARAAS ${ }^{(B)}$ IVIG $5 \%$ (human) manufactured by Shanghai RAAS packed by VACERA Egypt. This product is stabilized by sorbitol to avoid the osmotic insult induced by sucrose on proximal renal tubules ${ }^{(41)}$.

Finally, intravenous immunoglobulins are tools 
of an emerging technology in current medicine: immnoregulation.

The immune system has abandoned the status of mere champion of the body against infectious microbes and has assumed a position in the organic integration system with complex mechanisms essentially present in all diseases.

So far this is the only Egyptian RCT using IVIG in secondary RM.

Standardized IVIG regimens and more larger scaled placebo-controlled trials should be done to establish its use in RM.

\section{REFERENCES}

I- Regan L. (1988) : Prospective study of spontaneous abortion. In Beard, R.W and Sharp F. (eds). Early pregnancy loss : Mechanisms and treatment Springer- Verlag, London, pp.33-37.

2- Clifford K, Rai R, Waston $H$ et al (1994) : An informative protocol for the investigation of recurrent miscarriage: priliminary experience of 500 consecutive cases Hum. Reprod q,1328-1332.

3- Heine O. and Muller- Eckhardt G (1994): Intravenous immunoglobulin in recurrent abortion. Clin Exp. Immunol., 97, 39-42.

4- Ogasawara M.,Aaki K, Okada s, et al (2000): Embryonic Karytype of abortuses in relation to the number of pervious miscarriages Fertil Steril,, 73, 300-304.

5- Christiansen OB, Kilpatrick D.C, Souter V et al (1998): Mamman- binding lectin deficiency is associated with unexplained recurrent miscarriage, Scand j. Immunol ., 49,193-196.

6- Christiansen O.B, Rasmussen KL, Jersild C et al (1994): HLA Class II alleles confer susceptibility to recurrent fetal loss in Danish women. Tissue Antigens 44,225-233.

7- Pfeiffer KA, Fimmers R, Engels G et al(2001): The HLA-G genotype is potentially associated with idiopathic recurrent spontaneous abortion. Md. Hum. Reprod., 7, 373-378.
8- Petri M, Glbus M., Anderson R, Whiting-O'keefe Q, et al (1987): Antinuclear antibody lupus anticoagulant and cardiolipin antibody in woman with idiopathic habitual abortion Arthritis Rheum. $30,601-606$.

9- Xu L., Chang V., Murphy A, Rock J.A, Damewood $M$, Schlaff Wet al (1990): Antinuclear antibodies in sera of patients with recurrent pregnancy wastage. Am. Jobset. Gyneol . 163,1493-1497.

10- Hill J.A, Polgar K, Anderson D.J (1995): T helper 1-type immunity to trophoblast in women with recurrent spontaneous abortion. J.Am. Med. Assoc. 273,1933-1936.

11- Recurrent Miscarriage Immunotherapy Trialists Group (RMITG) (1994): Worldwide collaborative observational study and meta-analysis on allogenic leukocyte immunotherapy for recurrent spontaneous abortion. An.J. Reprod. Immunal. 32,55-72.

12- Daya S., Gunby J. RM IIG (1994): The effectiveness of allogenic lcukocyte immunization in unexplained primary recurrent spontaneous abortion. Am .J . Reprod. Immunol 32, 294-302.

13- Clynes R. and Raverch J.V. (1995): Cytotoxic antibodies trigger inflammation thought $\mathrm{Fc}$ receptors. Immunity 3:21-26.

14- Prasad N.K. papoff G., Zeuner A et al (1998): Therapeutic preparations of normal polyspecific IgG (IVIG) induce apoptosis in human lymphacytes and monocytes :a novel mechanism of action of IVIG involving the Fas apoptopic pathway J. immunal., 161,3781-3790.

15- Clark D. A, Caulam C.B (1996): Is there an immunological cause of recurrent pregnancy wastage ? Adv. Obstet Gynecol., 3, $321-342$.

16- Christiansen O.B, Mathiesen O., Husth $M$ etal (1995): Placebo-controlled trial of unexplained secondary recurrent spontaneous abortions and late spontaneous abortions with I.V immunglobulin Hum. Reprod., 10,2690-2695.

17- Coulam C.,Kysa L, Stern J et al (1995) : Intravenous immunoglobulin for treatment of recurrent pregnancy loss. AM. J Reprod. Immunol., 34, 333-337.

18- The German RSA/VIG group (1994): Intravenous immunoglobulin in the prevention of recurrent miscarriage. Br.J.Obstet. Gynecol. 101,1072-1077. 
19. Perino A., Vassiliadis A, Vucetich A. et al (1997): Short-term therapy for recurrent abortion using intravenous immunoglobulins: results of a double-blind placebo-controlled Italian study. Hum. Reprod., 12, 2388-2392.

20- Stephenson M.D, Dreher K, Houlihan E et al. (1998): Prevention of unexplained recurrent spontaneous abortion using intravenous immunoglobulin : a prospective, randomized double blinded, placebo- controlled trial, Am. J. Reprod. Immunol., 39, 82-88.

21- Jablonowska B., Selbing A, palfi M et al (1999); Prevention of recurrent spontaneous abortion by intravenous immunoglobalin: a double blind placebo-controlled study. Hum- Reprod, 14,838-841.

22- Christiansen O.B, Pederson B., Rosgaaed A. et al (2002): A randomized, double blind, placebocontrolled trial of intravenous immunoglobulin in the prevention of recurrent miscarriage : evidence for a therapeutic effect in woman with secondary recurrent miscarriage, Hum. Reprod 17(3) pp. 809-816.

23- Scolt J.R,(2003): Immunotherapy for recurrent miscarriage Cochrane Database Syst. Rev. 1, CDooolle.

24- Christiansen OB, Nielsen H.S. and Pedersen B. (2004): Active or passive immunization in unexplained recurrent miscarriage. J. Reprod-Imm. $62,41-52$.

25- Carreras LO, Perez GN, Vega HR et al (1988): Lupus anticoagulant and recurrent fetal loss; successful treatment with gammaglobulin jlancet ii, 393-394.

26- Thornton M. and Ballow (1993): Safety of intravenous immunoglobulin Arch Neurol So pp 135- 136 .

27- Bard F, Cannen C., Barbour R et al (2000): Peripherally administrated antibodies against amyloidy beta-peptide, Nat. Med 6:916-919.

28- Brekke OH, Loset GA. (2003): New technologies in therapeutic antibody development curr opin pharm 3:544-559.

29. Gura T. (2002): Therapeutic Antibodies Magic bullets hit the target . Nature 417:584-586.

30- Kwak J.y, Kwak FM, Ainbinder SM, et al (1996): Elevated peripheral blood natural killer cells are effectively suppressed by immunoglobulin G infusions in women with recurrent spontaneous abortions .AmJ. Reprod Immunol. 35,363-369.

31- Mueller- Eckhardt G., Heine O, et al (1989): prevention of recurrent spontaneous abortion by intravenous immunoglobulin. Vox. Sang 56, 151-154.

32- Christiansen OB. Mathicseno, Lauritsen $G$ et al (1992): Intravenous immunoglobulin treatment on women with multiple miscarriage Hum. Reprod 7 , 718-722.

33- Coulam C. B, Sten JJ. et al (1994) : Ultrasonagraphic findings of pregnancy losses aftcr treatment for recurrent pregnancy loss intravenous immunoglobulin versus placebo Fertil Steril 61, 248-251.

34- Christansen OB (1996): A fresh look at the causes and treatment of recurrent miscarriages, especially its immunological aspects Hum. Reprod. Update 2,271-293.

35- Kruse C. Rosgaard A, ct al (2002): Low scrum level of manna-binding lectin is a detorminant for pregnancy outcome in women with recurrent spontaneous abortion. Am.J. Obstet Gyncol. 187, 1313-1320.

36- Carp HJA, Ahiron R, Mashiachs et al (1996): Intravenous immunoglobulin in women with five or more abortions. Am j. Reprod. Immunol, 35, 360-362.

37- Yamada. H, Tatsurok, Kohayashi N.,(1998): Massive immunoglobulin treatment in women with four or more rccurrent spontaneous primary abortions of unexplained etiology, Hum. Reprod 13 (q):2620-23.

38- Samuclsson A; Tower T.L and Ravetch JV (2001): Anti-inflammatory activity of IVIG mediated through the inhibitory $\mathrm{Fc}$ receplor science. $291: 484-486$.

39- Colakoglu M.C Arslan E, Gezginek et al (2004): Serum TNF, IL-6 lupus anticoagulant and anticardiolupin antibody in women with and without a past history of recurrent miscarriage. Int. Congres series 1271 , sep.,50-53.

40- Ahsan N., Wicgand LA et al (1996): Acute renal failure following immunogbbulin therapy. Am.j. Nephrol, 16:532-6.

41- Kwan TH, Tong MKH, siu Yp, leug KT (2005): Acutc renal failure related to intravenous immunoglobulin infusion in an clderly woman. Hong Kong Med.J. 1 (1) feb pp.45-49. 